PROCEEDINGS OF THE

AMERICAN MATHEMATICAL SOCIETY

Volume 130, Number 11, Pages 3133-3139

S 0002-9939(02)06712-6

Article electronically published on May 29, 2002

\title{
NON-VANISHING OF SYMMETRIC SQUARE $L$-FUNCTIONS
}

\author{
YUK-KAM LAU
}

(Communicated by Dennis A. Hejhal)

\begin{abstract}
Given a complex number $s$ with $0<\Re e s<1$, we study the existence of a cusp form of large even weight for the full modular group such that its associated symmetric square $L$-function $L\left(\operatorname{sym}^{2} f, s\right)$ does not vanish. This problem is also considered in other articles.
\end{abstract}

\section{INTRODUCTION}

Let $k$ be an even positive integer and $f$ a holomorphic cusp form of weight $k$ with respect to the full modular group. We represent the Fourier expansion of $f$ (at the cusp $\infty$ ) by

$$
f(z)=\sum_{n=1}^{\infty} \psi_{f}(n) n^{(k-1) / 2} e(n z)
$$

where $e(\alpha)=e^{2 \pi i \alpha}$. Assume that $f(z)$ is an eigenfunction for all Hecke operators $T_{n}$, with $T_{n} f=\lambda_{f}(n) n^{(k-1) / 2} f$. Note that $\lambda_{f}(n)$ is real and has the Deligne's bound

$$
\left|\lambda_{f}(n)\right| \leq \tau(n)
$$

where $\tau(n)=\sum_{d \mid n} 1$ is the divisor function. We normalize $f$ so that $\psi_{f}(1)=1$; then we have $\psi_{f}(n)=\lambda_{f}(n)$. Such an $f$ is called a primitive form. Associated to each primitive $f$, the Rankin-Selberg convolution $L$-function $L(f \otimes f, s)$ and the symmetric square $L$-function $L\left(\operatorname{sym}^{2} f, s\right)$ are respectively defined as, for $\Re e s>1$,

$$
L(f \otimes f, s)=\sum_{n=1}^{\infty} \lambda_{f}(n)^{2} n^{-s}
$$

and

$$
L\left(\operatorname{sym}^{2} f, s\right)=\zeta(2 s) \sum_{n=1}^{\infty} \lambda_{f}\left(n^{2}\right) n^{-s}
$$

where $\zeta(s)$ is the Riemann zeta-function. These two $L$-functions are closely linked by the relation (see [5, (0.2) and (0.4)])

$$
\zeta(s) L\left(\operatorname{sym}^{2} f, s\right)=\zeta(2 s) L(f \otimes f, s) .
$$

In this paper, we are concerned with the non-vanishing results of $L\left(\operatorname{sym}^{2} f, s\right)$ in the critical strip. $\mathrm{Li}$ [4] showed that for a given complex number $\rho \neq 1 / 2$

Received by the editors February 6, 2001.

2000 Mathematics Subject Classification. Primary 11F66.

(C)2002 American Mathematical Society 
satisfying $0<\Re e \rho<1$ and $\zeta(\rho) \neq 0$, there are infinitely many primitive forms $f$ of different weight such that $\zeta(2 s) L(f \otimes f, s)$ do not vanish at $s=\rho$, or equivalently, $L\left(\operatorname{sym}^{2} f, \rho\right) \neq 0$. In addition, Kohnen and Sengutpa 3 have recently showed that for any fixed $s=\sigma+i t$ with $0<\sigma<1$ and $\sigma \neq 1 / 2$, and for all sufficiently large $k$, there exists a primitive form $f$ of weight $k$ such that $L\left(\operatorname{sym}^{2} f, s\right) \neq 0$. The approaches used in [4] and 3] are different: the former utilizes an approximate functional equation for an averaged sum of $L\left(\operatorname{sym}^{2} f, \rho\right)$ while the latter relies on a formula of Zagier. Here, we shall use another method to prove the theorem below, which includes the results in [3] and [4].

Theorem. For any fixed $s \in \mathbf{C}$ with $0<\Re e s<1$, there exist infinitely many even $k$ such that $L\left(\operatorname{sym}^{2} f, s\right) \neq 0$ for some primitive form $f$ of weight $k$. Furthermore, when $\Re e s \neq 1 / 2$ or $s=1 / 2$, there exists a constant $k_{0}(s)$ depending on $s$ such that for all even $k \geq k_{0}(s), L\left(s^{2} f, s\right)$ does not vanish for some primitive form $f$ of weight $k$.

Remark. The case $s=1 / 2$ is not treated in either [3] or [4]. Moreover, our alternative proof is somewhat simpler than [4], and seems more 'elementary' than [3] (without using Zagier's formula).

\section{Preliminaries}

Let $S_{k}(1)$ be the linear space of cusp forms of weight $k$ for the full modular group $\Gamma=S L_{2}(\mathbf{Z})$. Then $S_{k}(1)$ is a finite-dimensional Hilbert space with respect to the Petersson inner product

$$
\langle f, g\rangle=\int_{\Gamma \backslash \mathbf{H}} y^{k} f(z) \overline{g(z)} \frac{d x d y}{y^{2}}
$$

and the set of all primitive forms $\mathcal{B}_{k}$ forms an orthogonal basis for $S_{k}(1)$. Moreover, we have the Petersson trace formula: define

$$
w_{f}=\frac{\Gamma(k-1)}{(4 \pi)^{k-1}\langle f, f\rangle}
$$

and $S(m, n, c)=\sum_{a d \equiv 1(c)} e((a m+d n) / c)$ (the classical Kloosterman sum); then

$$
\sum_{f \in \mathcal{B}_{k}} w_{f} \lambda_{f}(m) \lambda_{f}(n)=\delta_{m, n}+2 \pi i^{-k} \sum_{c \geq 1} c^{-1} S(m, n, c) J_{k-1}\left(\frac{4 \pi \sqrt{m n}}{c}\right)
$$

where $\delta_{m, n}=1$ or 0 according to whether $m=n$ or not, and $J_{k-1}(x)$ is the Bessel function. From [6] (5) in Section 2.13], we have the integral representation

$$
J_{k-1}(x)=\frac{1}{2 \pi} \int_{-\pi}^{\pi} e^{-i(k-1) \theta+i x \sin \theta} d \theta .
$$

Bounding trivially, using integration by parts or the Poisson integral representation $J_{k-1}(x)=(\sqrt{\pi} \Gamma(k-1 / 2))^{-1}(x / 2)^{k-1} \int_{-1}^{1}\left(1-t^{2}\right)^{k-3 / 2} e^{i x t} d t$ ([]. (3) in 2.3]), we have the following estimates: for $x \geq 0$,
(i) $J_{k-1}(x) \ll 1$,
(ii) $J_{k-1}(x) \ll \frac{x}{k}$,
(iii) $J_{k-1}(x) \ll \frac{1}{\Gamma(k-1 / 2)}\left(\frac{x}{2}\right)^{k-1}$. 
Using the Weil bound

$$
|S(m, n, c)| \leq(m, n, c)^{1 / 2} c^{1 / 2} \tau(c),
$$

and $\lambda_{f}(1)=1$, we have with (2.3) (ii)

$$
\sum_{f \in \mathcal{B}_{k}} w_{f} \ll 1+k^{-1} \sum_{c \geq 1} c^{-3 / 2} \tau(c) \ll 1 .
$$

Define

$$
\begin{aligned}
\Delta(s) & =\pi^{-3 s / 2} \Gamma\left(\frac{s+1}{2}\right) \Gamma\left(\frac{s+k-1}{2}\right) \Gamma\left(\frac{s+k}{2}\right) \\
& =\pi^{(1-3 s) / 2} 2^{1-s-k} \Gamma(s+k-1) \Gamma\left(\frac{s+1}{2}\right)
\end{aligned}
$$

(as $\left.\Gamma(s) \Gamma(s+1 / 2)=\sqrt{\pi} 2^{1-2 s} \Gamma(2 s)\right)$ and $\Lambda\left(\operatorname{sym}^{2} f, s\right)=\Delta(s) L\left(\operatorname{sym}^{2} f, s\right)$. Then $\Lambda\left(\operatorname{sym}^{2} f, s\right)$ is entire and satisfies the functional equation (shown by Shimura [5])

$$
\Lambda\left(\operatorname{sym}^{2} f, s\right)=\Lambda\left(\operatorname{sym}^{2} f, 1-s\right) .
$$

Moreover one can show that $\Lambda\left(\operatorname{sym}^{2} f, s\right) \rightarrow 0$ as $|\operatorname{Im} s| \rightarrow \infty$ in any vertical strip $|\Re e s| \ll 1$.

Finally, let us explain the approach here (which is quite widely used in nonvanishing problems). Using residue theorem and the functional equation of $L\left(\operatorname{sym}^{2} f, \cdot\right)$, we can express $L\left(\operatorname{sym}^{2} f, s\right)$ as a convergent series. The averaging process (over all primitive forms) with Petersson trace formula yields that the (averaged) sum consists of two parts: the diagonal terms (contributed by $\delta_{m, n}$ in (2.1) and the off-diagonal terms. (See (3.6) below.) We then obtain the asymptotic formula (3.13) after giving an estimation to the off-diagonal terms. Our result is deduced from this formula.

\section{Proof of the Theorem}

Assume $0<\Re e s \leq 1 / 2$. Consider the integral $(2 \pi i)^{-1} \int_{\mathcal{R}} \Lambda\left(\operatorname{sym}^{2} f, s+w\right) d w / w$ where $\mathcal{R}$ is the positively oriented rectangular contour with vertices at $\pm 2 \pm i T$, we have, by residue theorem and taking $T \rightarrow \infty$, that

$$
\begin{aligned}
\Lambda\left(\operatorname{sym}^{2} f, s\right) & =\frac{1}{2 \pi i}\left(\int_{(2)}-\int_{(-2)}\right) \Lambda\left(\operatorname{sym}^{2} f, s+w\right) \frac{d w}{w} \\
& =\frac{1}{2 \pi i} \int_{(2)} \Lambda\left(\operatorname{sym}^{2} f, s+w\right) \frac{d w}{w}+\frac{1}{2 \pi i} \int_{(2)} \Lambda\left(\operatorname{sym}^{2} f, 1-s+w\right) \frac{d w}{w}
\end{aligned}
$$

after using the functional equation (2.7) and changing $w$ to $-w$. Hence, if we write

$$
V_{z}(y)=\frac{1}{2 \pi i} \int_{(2)} \zeta(2(z+w)) \Delta(z+w) y^{-w} \frac{d w}{w},
$$

we get from (1.2) that

$$
L\left(\operatorname{sym}^{2} f, s\right) \Delta(s)=\sum_{n=1}^{\infty} \frac{\lambda_{f}\left(n^{2}\right)}{n^{1-s}} V_{1-s}(n)+\sum_{n=1}^{\infty} \frac{\lambda_{f}\left(n^{2}\right)}{n^{s}} V_{s}(n) .
$$

Let $z=1-s$ or $s$. From (2.6),

$$
\Delta(z+w) \ll_{|s|} 2^{-k} \Gamma(\Re e(z+w)+k-1)\left|\Gamma\left(\frac{z+w+1}{2}\right)\right|
$$


for $\Re e(z+w) \geq-3 / 4$. Moving the line of integration to $\Re e(z+w)=A$, we have for $A>\max (\Re e z, 1 / 2)$,

$$
V_{z}(y) \ll_{|s|, A} y^{\Re e z-A} 2^{-k} \Gamma(k+A-1) .
$$

Shifting to $\Re e(z+w)=-1 / 2$ (across the poles at $w=0,1 / 2-z)$, we obtain with (3.3)

$$
V_{z}(1)=\left\{\begin{array}{l}
\zeta(2 z) \Delta(z)+\Delta(1 / 2)(1 / 2-z)^{-1} \\
\gamma \Delta(1 / 2)+2^{-1} \Delta^{\prime}(1 / 2)
\end{array}+O\left(2^{-k} \Gamma(k-3 / 2)\right)\right.
$$

where $\gamma$ is the Euler constant. The second case corresponds to $z=1 / 2$. As will be seen, the main term is given by

$$
V_{1-s}(1)+V_{s}(1)=\left\{\begin{array}{l}
\zeta(2-2 s) \Delta(1-s)+\zeta(2 s) \Delta(s) \\
2 \gamma \Delta(1 / 2)+\Delta^{\prime}(1 / 2)
\end{array}+\cdots\right.
$$

according to $s \neq 1 / 2$ or $s=1 / 2$. Its order of magnitude is about $2^{-k} \Gamma(k-\Re e s)$.

Let $0<\nu \leq 10^{-3}$ be a fixed number. Both sums in (B.2) over $n>k^{1+5 \nu}$ can be evaluated as follows: choosing $A=1+\nu^{-1}$ in (3.4), we have $(z=1-s$ or $s)$

$$
\begin{aligned}
\sum_{n>k^{1+5 \nu}} \frac{\lambda_{f}\left(n^{2}\right)}{n^{z}} V_{z}(n) & \ll 2^{-k} \Gamma\left(k+\nu^{-1}\right) \sum_{n>k^{1+5 \nu}} \frac{\tau\left(n^{2}\right)}{n^{1+1 / \nu}} \\
& \ll 2^{-k} k^{-4-1 / \nu} \Gamma\left(k+\nu^{-1}\right) \ll 2^{-k} k^{-1 / 4} \Gamma(k-1 / 2)
\end{aligned}
$$

by (1.1) and Stirling's formula ([1, Chapter 10]). Summing over all primitive forms and using (2.1), with $\lambda_{f}(1)=1$,

$$
\begin{aligned}
& \Delta(s) \sum_{f \in \mathcal{B}_{k}} w_{f} L\left(\operatorname{sym}^{2} f, s\right) \\
& =V_{1-s}(1)+V_{s}(1)+\sum_{z=1-s, s} 2 \pi i^{-k} \sum_{n \leq k^{1+5}} n^{-z} V_{z}(n) \mathcal{J}(n) \\
& \quad+O\left(\left(\sum_{f} w_{f}\right) 2^{-k} k^{-1 / 4} \Gamma(k-1 / 2)\right)
\end{aligned}
$$

where $\mathcal{J}(n)=\sum_{c>1} c^{-1} S\left(1, n^{2}, c\right) J_{k-1}(4 \pi n / c)$. We give an estimate for $\mathcal{J}(n)$. From (2.3)(ii) and (2.4),

$$
\sum_{c>k^{1+20 \nu}} c^{-1} S\left(1, n^{2}, c\right) J_{k-1}\left(\frac{4 \pi n}{c}\right) \ll n k^{-1} \sum_{c>k^{1+20 \nu}} c^{-3 / 2} \tau(c) \ll n / k^{3 / 2+9 \nu} .
$$

By (2.3) (iii), when $n \leq k^{1-\nu}$,

$$
\begin{aligned}
\sum_{c \leq k^{1+20 \nu}} c^{-1} S\left(1, n^{2}, c\right) J_{k-1}\left(\frac{4 \pi n}{c}\right) & \ll \Gamma(k-1 / 2)^{-1} \sum_{c \leq k^{1+20 \nu}} c^{-1 / 2} \tau(c)\left(2 \pi k^{1-\nu}\right)^{k-1} \\
& \ll k^{(1-\nu) k} \Gamma(k-1 / 2)^{-1} \ll n / k^{3 / 2+9 \nu},
\end{aligned}
$$

by Stirling's formula. Similarly, for $k^{1-\nu}<n \leq k^{1+5 \nu}$ we have

$$
\sum_{k^{6 \nu}<c \leq k^{1+20 \nu}} c^{-1} S\left(1, n^{2}, c\right) J_{k-1}(4 \pi n / c) \ll n / k^{3 / 2+9 \nu} .
$$


Hence,

$$
\mathcal{J}(n)=\delta(n, k) \sum_{c \leq k^{6 \nu}} c^{-1} S\left(1, n^{2}, c\right) J_{k-1}\left(\frac{4 \pi n}{c}\right)+O\left(n k^{-3 / 2-9 \nu}\right)
$$

where $\delta(n, k)=0$ if $n \leq k^{1-\nu}$, and 1 if $k^{1-\nu}<n \leq k^{1+5 \nu}$. Inserting (3.7) into (3.6), together with (2.5) and the estimate

$k^{-3 / 2-9 \nu} \sum_{n \leq k^{1+5 \nu}}\left|n^{1-z} V_{z}(n)\right| \ll 2^{-k} k^{-3 / 2-9 \nu}(\log k) \Gamma(k+1) \ll 2^{-k} k^{-2 \nu} \Gamma(k-1 / 2)$

(following from (3.4) with $A=2$ ), we see that (3.6) becomes

$$
\begin{aligned}
& \Delta(s) \sum_{f} w_{f} L\left(\operatorname{sym}^{2} f, s\right) \\
= & V_{1-s}(1)+V_{s}(1)+i^{-k}\left(\mathcal{E}_{k}(1-s)+\mathcal{E}_{k}(s)\right)+O\left(2^{-k} k^{-8 \nu} \Gamma(k-1 / 2)\right)
\end{aligned}
$$

where

$$
\mathcal{E}_{k}(z)=2 \pi \sum_{k^{1-\nu}<n \leq k^{1+5 \nu}} n^{-z} V_{z}(n) \sum_{c \leq k^{6 \nu}} c^{-1} S\left(1, n^{2}, c\right) J_{k-1}\left(\frac{4 \pi n}{c}\right) .
$$

From (2.2), we have

$$
\mathcal{E}_{k}(z)=\sum_{k^{1-\nu}<n \leq k^{1+5 \nu}} n^{-z} V_{z}(n) \sum_{c \leq k^{6 \nu}} c^{-1} S\left(1, n^{2}, c\right) \int_{0}^{\pi / 2} 2 \Re e f_{k}\left(\theta, \frac{4 \pi n}{c}\right) d \theta
$$

where $f_{k}(\theta, x)=e^{i x \sin \theta}\left(e^{-i(k-1) \theta}-e^{i(k-1) \theta}\right)$. When $|x| \leq k^{6 / 5}$, we have

$$
\left|\frac{d}{d \theta}(x \sin \theta \pm(k-1) \theta)\right| \asymp k \quad \text { for } \pi / 2-k^{-1 / 4} \leq \theta \leq \pi / 2,
$$

whence $\int_{\pi / 2-k^{-1 / 4}}^{\pi / 2} f_{k}\left(\theta, \frac{4 \pi n}{c}\right) d \theta \ll k^{-1}$ for $4 \pi n / c \leq k^{6 / 5}$ by integration by parts. From (3.4) with $A=1$ and (2.4),

$$
\begin{aligned}
& \sum_{k^{1-\nu}<n \leq k^{1+5 \nu}} n^{-z} V_{z}(n) \sum_{c \leq k^{6 \nu}} c^{-1} S\left(1, n^{2}, c\right) \int_{\pi / 2-k^{-1 / 4}}^{\pi / 2} \Re e f_{k}\left(\theta, \frac{4 \pi n}{c}\right) d \theta \\
\ll & 2^{-k} k^{-1} \Gamma(k) \sum_{k^{1-\nu}<n \leq k^{1+5 \nu}} n^{-1} \sum_{c \leq k^{6 \nu}} c^{-1 / 2} \tau(c) \ll 2^{-k} k^{-8 \nu} \Gamma(k-1 / 2) .
\end{aligned}
$$

We put this estimate into (3.9). Then we interchange the sums in the remaining part and use the periodicity of $S(1, \cdot, c)$ to give

$$
\begin{aligned}
\mathcal{E}_{k}(z)= & \sum_{c \leq k^{6 \nu}} c^{-1} \sum_{k^{1-\nu}<n \leq k^{1+5 \nu}} S\left(1, n^{2}, c\right) n^{-z} V_{z}(n) \int_{0}^{\pi / 2-k^{-1 / 4}} 2 \Re e f_{k}\left(\theta, \frac{4 \pi n}{c}\right) d \theta \\
& +O\left(2^{-k} k^{-8 \nu} \Gamma(k-1 / 2)\right) \\
= & \sum_{c \leq k^{6 \nu}} \sum_{0 \leq r<c} c^{-1} S\left(1, r^{2}, c\right) T_{z}(r, c)+O\left(2^{-k} k^{-8 \nu} \Gamma(k-1 / 2)\right)
\end{aligned}
$$


with

$$
T_{z}(r, c)=2 \sum_{\substack{1-\nu<n \leq k 1+5 \nu \\ n \equiv r(c)}} n^{-z} V_{z}(n) \int_{0}^{\pi / 2-k^{-1 / 4}} \Re e f_{k}\left(\theta, \frac{4 \pi n}{c}\right) d \theta .
$$

From the definition of $f_{k}(\theta, \cdot)$ (the line below (3.9)), we see that

$$
\begin{aligned}
& T_{z}(r, c) \ll \int_{0}^{\pi / 2-k^{-1 / 4}}\left|\sum_{\substack{k^{1-\nu}<n \leq k 1+5 \nu \\
n \equiv r(c)}} n^{-z} V_{z}(n) e\left(\frac{2 n}{c} \sin \theta\right)\right| d \theta \\
& =\int_{0}^{\pi / 2-k^{-1 / 4}}\left|\int_{(\kappa)} \zeta(2(z+w)) \Delta(z+w) \sum_{\substack{1-\nu<n \leq k 1+5 \nu \\
k \equiv k^{1}(c)}} n^{-z-w} e\left(\frac{2 n}{c} \sin \theta\right) \frac{d w}{w}\right| d \theta
\end{aligned}
$$

by (3.1) with the path moved from $\Re e w=2$ to $\kappa=2-\Re e z$. By (3.3), $\Delta(z+w) \ll$ $2^{-k} \Gamma(k+1)(|w|+1)^{-2}$ for $\Re e w=\kappa$. Hence,

$$
\begin{aligned}
T_{z}(r, c) \ll & 2^{-k} \Gamma(k+1) \\
& \times \int_{(\kappa)} \int_{0}^{\pi / 2-k^{-1 / 4}}\left|\sum_{K_{1}<m \leq K_{2}} \frac{e(2 m \sin \theta)}{(c m+r)^{z+w}}\right| d \theta \frac{|d w|}{(|w|+1)^{3}}
\end{aligned}
$$

where $K_{1}=\left(k^{1-\nu}-r\right) / c$ and $K_{2}=\left(k^{1+5 \nu}-r\right) / c$. Using $\sum_{m \leq M} e(2 m \alpha)$ $\ll|\sin (2 \pi \alpha)|^{-1}$ with partial summation, or bounding trivially, the sum in (3.11) is

$$
\ll(|w|+1) k^{2 \nu-2} \min \left(|\sin (2 \pi \sin \theta)|^{-1}, k\right)
$$

as $\Re e(z+w)=2$. After substituting (3.12) into (3.11), the $\theta$-integral equals

$$
\begin{aligned}
& \int_{0}^{\pi / 2-k^{-1 / 4}} \min \left(|\sin (2 \pi \sin \theta)|^{-1}, k\right) d \theta \\
= & \int_{0}^{\cos \left(k^{-1 / 4}\right)} \min \left(|\sin (2 \pi u)|^{-1}, k\right) \frac{d u}{\sqrt{1-u^{2}}} \\
\ll & \left(\int_{0}^{k^{-1}}+\int_{1 / 2-k^{-1}}^{1 / 2+k^{-1}}\right) k d u+\left(\int_{k^{-1}}^{1 / 2-k^{-1}}+\int_{1 / 2+k^{-1}}^{3 / 4}\right)|\sin (2 \pi u)|^{-1} d u \\
& +\int_{3 / 4}^{1-(16 k)^{-1 / 2}}|\sin (2 \pi u)|^{-1} \frac{d u}{\sqrt{1-u}} \ll 1+\log k+k^{1 / 4}
\end{aligned}
$$

by using $\sin \alpha \geq 2 \alpha / \pi$ if $0 \leq \alpha \leq \pi / 2$. In view of (3.11) and (3.12), we conclude that $T_{z}(r, c) \ll 2^{-k} k^{2 \nu-7 / 4} \Gamma(k+1) \ll 2^{-k} k^{2 \nu-1 / 4} \Gamma(k-1 / 2)$, and by (3.10) that

$$
\mathcal{E}_{k}(z) \ll 2^{-k} k^{2 \nu-1 / 4} \Gamma(k-1 / 2) \sum_{c \leq k^{6 \nu}} \sum_{0 \leq r<c} c^{-1}\left|S\left(1, r^{2}, c\right)\right|+2^{-k} k^{-8 \nu} \Gamma(k-1 / 2)
$$

which is absorbed by the $O$-term in (3.8). Therefore, (3.8) and (3.5) yield

$$
\begin{aligned}
& \Delta(s) \sum_{f \in \mathcal{B}_{k}} w_{f} L\left(\mathrm{sym}^{2} f, s\right) \\
= & \left\{\begin{array}{l}
\zeta(2-2 s) \Delta(1-s)+\zeta(2 s) \Delta(s) \\
\Delta^{\prime}(1 / 2)+2 \gamma \Delta(1 / 2)
\end{array}+O\left(2^{-k} k^{-8 \nu} \Gamma(k-1 / 2)\right) .\right.
\end{aligned}
$$


From Stirling's formula, we have $\Gamma(k+z-1)=\Gamma(k+a-1) e^{i b \log k+O(1 / k)}$ $(z=a+i b)$ for $|z| \leq k^{1 / 3}$ and $\Gamma^{\prime}(k-1 / 2) / \Gamma(k-1 / 2)=\log k+O(1)$. Hence for the case $s=1 / 2$, the dominating term in (3.13) is $\Delta^{\prime}(1 / 2)$, of order $2^{-k}(\log k) \Gamma(k-1 / 2)$, for all large $k$, and we can thus conclude $\sum_{f \in \mathcal{B}_{k}} w_{f} L\left(\operatorname{sym}^{2} f, 1 / 2\right) \neq 0$. For the case $\Re e s<1 / 2$, the term $\zeta(2-2 s) \Delta(1-s)\left(\asymp 2^{-k} \Gamma(k-\Re e s)\right)$ dominates others for all large $k$. (Note that $\zeta(2-2 s)$ is non-zero.) When $s=1 / 2+i t$ and $t \neq 0$, denoting $a(t)=2^{1 / 2-i t} \pi^{-1 / 4-3 i t / 2} \zeta(1+2 i t) \Gamma(3 / 4+i t / 2)$, the main term in (3.13) is

$$
\begin{aligned}
& \zeta(1+2 i t) \Delta(1 / 2+i t)+\zeta(1-2 i t) \Delta(1 / 2-i t) \\
= & 2^{-k}(a(t) \Gamma(k-1 / 2+i t)+a(-t) \Gamma(k-1 / 2-i t)) \\
= & 2^{-k} \Gamma(k-1 / 2)\left(2|a(t)| \cos (t \log k+\vartheta(t))+O\left(k^{-1}\right)\right)
\end{aligned}
$$

where $\vartheta(t)$ is the argument of $a(t)$. Suppose $(2 \pi)^{-1} t \log 2$ is irrational. Then by Kronecker's theorem ([2, Theorem 438]), there exist infinitely many $r_{i}$ (depending on $t$ ) satisfying $\left|r_{i} t \log 2+\vartheta(t)-2 \pi m_{i}\right| \leq \pi / 4$ for some integer $m_{i}$. Thus, we take $k=2^{r_{i}}$ for those sufficiently large $r_{i}$ so that the right side of (3.13) is $\gg$ $2^{-k}|a(t)| \Gamma(k-1 / 2)>0$. If $(2 \pi)^{-1} t \log 2$ is rational, we consider instead $(2 \pi)^{-1} t \log 3$ which must then be irrational. Our result follows with the previous argument. The case $1 / 2<\Re e s<1$ is done because of the functional equation (2.7).

\section{ACKNOWLEDGEMENTS}

The author expresses his sincere gratitude to the referee for the valuable comments. He also thanks Dr. Kai-Man Tsang for his encouragements and Charlies Tu for unfailing supports.

\section{REFERENCES}

[1] H. Davenport, Multiplicative Number Theory, Second edition, Springer-Verlag, 1980. MR 82m:10001

[2] G.H. Hardy and E.M. Wright, An Introduction to the Theory of Numbers, Fifth edition, Oxford University Press, 1979. MR 81i:10002

[3] W. Kohnen and J. Sengupta, Nonvanishing of symmetric square L-functions of cusp forms inside the critical strip, Proc. Amer. Math. Soc. 128 (2000), 1641-1646. MR 2000j:11072

[4] X.-J. Li, On the poles of Rankin-Selberg convolution of modular forms, Trans. Amer. Math. Soc. 348 (1996), 1213-1234. MR 96h:11038

[5] G. Shimura, On the holomorphy of certain Dirichlet series, Proc. London Math. Soc. 31 (1975), 79-98. MR 52:3064

[6] G.N. Watson, A Treatise on the Theory of Bessel Function, Reprint, Cambridge University Press, 1996. MR 96i:33010

Institut Élie Cartan, Université Henri Poincaré (Nancy 1), 54506 Vandoeuvre lés Nancy Cedex, France

Current address: Department of Mathematics, The University of Hong Kong, Pokfulam Road, Hong Kong

E-mail address: yklau@maths.hku.hk 